\section{Affibody vs. amyloid}

\section{By Tim Fulmer, Senior Writer}

Swedish and British researchers have shown that Affibody AB's Affibodies can be engineered to bind and clear toxic $\beta$-amyloid from the brains of fruit flies. ${ }^{1}$ The academics are now planning to look at the Affibodies in rodent models of Alzheimer's disease.

The biotech is helping by increasing the bioavailability of its lead Affibody for $\mathrm{AD}, \mathrm{Z}_{\mathrm{A} \beta 3}$, but its focus remains on Affibodies for cancer.

High levels of $\beta$-amyloid $(A \beta)$ peptide in the brain, from either overproduction of the peptide or failure to clear it, are thought to lead to widespread $A \beta$ aggregates and plaques. These in turn cause neuronal and synaptic dysfunction and, ultimately, learning and memory deficits.

Because excessive $A \beta$ sits at the top of the amyloid cascade, one strategy has been to target the peptide and prevent it from forming the oligomers and aggregates that trigger CNS dysfunction. There are at least 10 antibody and small molecule inhibitors of $A \beta$ aggregates in the clinic to treat $A D$.

Affibodies represent a third option, offering a lower risk of immunogenicity than antibodies and better binding affinity than small molecules. Although they are less than half the size of an antibody, Affibodies still share the latter's strong binding affinity and stability.

Previously, structural biology professor Torleif Härd at the Swedish University of Agricultural Sciences, colleagues at the KTH Royal Institute of Technology and researchers at Affibody AB had identified an Affibody dimer that bound monomeric $A \beta$ with nanomolar affinity in vitro. ${ }^{2}$ Denoted $\mathrm{Z}_{\mathrm{A} \beta 3}$, the dimer consists of two covalently linked 58-amino-acid Affibody monomers and binds $\mathrm{A} \beta$ in a one-to-one ratio.

The next step was to see whether the binding affinity of $\mathrm{Z}_{\mathrm{A} \beta 3}$ translated into increased clearance and degradation of $\mathrm{A} \beta$ in an $\mathrm{AD}$ model. To do that, Härd collaborated with Christopher Dobson, a professor of chemistry whose lab at the University of Cambridge had created a Drosophila model of $\mathrm{AD}$ that expresses the toxic human form of $\mathrm{A} \beta$ in the brain.

By studying $\mathrm{Z}_{\mathrm{A} \beta 3}$ in flies before mice, the researchers hoped to get a better understanding of the Affibody's mechanism of action without having to deal with bioavailability and other pharmacokinetics issues that could have complicated the picture.

In flies with toxic human $A \beta$ in their brains, transgenic expression of $Z_{A \beta 3}$ improved survival compared with expression of a nonspecific mock Affibody (31 days vs. 9 days). Normal wild-type flies lived about 38 days.
Analysis of fly brain extracts showed that insects expressing $Z_{A \beta 3}$ in the presence of toxic $A \beta$ had a $97 \%$ reduction in $A \beta$ levels compared with flies expressing mock Affibody in the presence of toxic $A \beta$ $(p<0.01)$.

Thus, $\mathrm{Z}_{\mathrm{A} \beta 3}$ increased fly survival at least partly by increasing clearance of neurotoxic $A \beta$ from the brain.

Finally, in vitro binding studies gave some additional insight into mechanism. $Z_{A \beta 3}$ inhibited the formation of $A \beta$ fibrils by binding and sequestering the hydrophobic, aggregation-inducing regions of soluble monomeric $A \beta$.

The study, which was published in PLoS Biology, was led by Härd and Dobson.

Andrew Doig, professor of biochemistry at The University of Manchester and cofounder of Senexis Ltd., said a key question to answer will be whether targeting $A \beta$ with $Z_{A \beta 3}$ actually prevents cognitive decline.

"Even before going into animals, the researchers could see if the Affibody reverses inhibition of long-term potentiation by $\mathrm{A} \beta$ in a brain slice," he suggested. Long-term potentiation (LTP) is a long-term enhancement in signal transmission between neurons and is considered an important cellular mechanism that underlies learning and memory.

Doig is focused on small peptide inhibitors of $\mathrm{A} \beta$ aggregation called meptides. ${ }^{3}$
-Andrew Doig,
With the fly data in hand, Härd told SciBX it is now time to study $\mathrm{Z}_{\mathrm{A} \beta 3}$ in mouse models that more closely resemble human AD. That work will be carried out in collaboration with Stefan Ståhl, professor of molecular biotechnology at the Royal Institute of Technology, and Lars Abrahmsén, CSO of Affibody AB.

The labs of Härd and Ståhl will carry out the animal studies, whereas the company, which owns the IP covering the Affibody molecules, will focus on ways to modify $\mathrm{Z}_{\mathrm{A} \beta 3}$ to ensure the compound has good bioavailability.

\section{CNS vs. periphery}

The main goal will be to ensure that there are enough Affibodies in the serum to sop up $A \beta$.

$\mathrm{A} \beta$ concentrations in the CNS and the blood exist in dynamic equilibrium. As a result, it may not be necessary to get the Affibody across the blood brain barrier (BBB) for it to prevent amyloid plaque formation. By sequestering $A \beta$ monomers in the periphery, Affibody treatment should lower the number of monomers available to the CNS and thus could reduce formation of toxic amyloid aggregates in the brain.

Affibody AB plans to design an Affibody fusion protein consisting of $Z_{A \beta 3}$ linked to a peptide domain that reversibly binds serum albumin, thus prolonging the half-life of the compound.

The increased half-life should, in turn, "enhance the number of A $\beta$ monomers a given Affibody can sequester and thus allow for less fre- 


\section{ANALYSIS}

\section{TARGETS \& MECHANISMS}

quent dosing and lower serum concentrations of drug, which ultimately means reduced risk of off-target toxicities," Abrahmsén told SciBX.

Affibody $\mathrm{AB}$ already has proof of principle for the albumin-binding domain. In mouse xenografts, an anti-HER2 (ERBB2; neu) Affibody fused to an albumin-binding domain had 25-fold lower plasma clearance than a nonfused Affibody. ${ }^{4}$

Nonetheless, Senexis believes that because A $\beta$ aggregates cause toxicity only in the brain, a better strategy might be to target aggregation inhibitors directly to the CNS rather than have them work from a distance in the periphery. For that, protein-based therapeutics like antibodies, peptides and Affibodies could be less than ideal.

"Peptides designed to target $A \beta$ are very useful for mechanistic studies of amyloid aggregation pathways," said Senexis CEO J. Mark Treherne. "However, we believe that difficulties associated with bioavailability and penetration of the blood brain barrier make them less than ideal therapeutic candidates compared with orally available, CNS-penetrant small molecule aggregation inhibitors" such as the company's lead compound, SEN1500, which is in preclinical development for AD.

Treherne acknowledged that targeting A $\beta$ monomers primarily in the periphery could have therapeutic value. However, he said, "because the majority of $A \beta$ oligomers and toxic aggregates are clearly in the brain, we believe that is probably the main site of action for any $\mathrm{AD}$ therapeutic. It is therefore important that an aggregation inhibitor be able to cross the blood brain barrier in reasonably high concentrations."

To that end, Affibody $\mathrm{AB}$ and Härd also will consider ways to ferry
Affibodies across the BBB. Although targeting A $\beta$ in the periphery is technically more straightforward, there are likely means of achieving transport of an Affibody across the BBB, Abrahmsén said. He declined to disclose details.

Affibody continues to focus its efforts on cancer therapeutics. The biotech's lead compound is molecular imaging agent ABY-025, which is based on a HER2-specific Affibody. In 2009, ABY-025 received approval to begin a Phase I study in bladder cancer. The company did not disclose when the trial will begin.

Fulmer, T. SciBX 3(13); doi:10.1038/scibx.2010.397

Published online April 1, 2010

\section{REFERENCES}

1. Luheshi, L. et al. PLoS Biol.; published online March 16, 2010; doi:10.1371/journal.pbio.1000334

Contact: Torleif Härd, Swedish University of Agricultural Sciences, Uppsala, Sweden

e-mail: torleif.hard@molbio.slu.se

Contact: Christopher M. Dobson, University of Cambridge, Cambridge,

U.K.

e-mail: cmd44@cam.ac.uk

2. Hoyer, W. et al. Proc. Natl. Acad. Sci. USA 105, 5099-5104 (2008)

3. Kokkoni, N. et al. Biochemistry 45, 9906-9918 (2006)

4. Tolmachev, V. et al. Cancer Res. 67, 2773-2782 (2007)

COMPANIES AND INSTITUTIONS MENTIONED

Affibody AB, Stockholm, Sweden

KTH Royal Institute of Technology, Stockholm, Sweden

Senexis Ltd., Cambridge, U.K.

Swedish University of Agricultural Sciences, Uppsala, Sweden

University of Cambridge, Cambridge, U.K.

The University of Manchester, Manchester, U.K. 